\title{
Falx Cerebelli Meningioma: Case Report of a Rarely Occurring Tumor, Management Nuances, and Literature Review
}

\author{
Elizabeth Gallo ${ }^{1}$ Grzegorz Brzezicki ${ }^{1}$ Raafat Makary ${ }^{1}$ Gazanfar Rahmathulla ${ }^{1}$ Dinesh Rao ${ }^{1}$ \\ Daryoush Tavanaiepour ${ }^{1}$
} ${ }^{1}$ Department of Neurosurgery, University of Florida, College of
Medicine, Jacksonville, Florida, United Sates

J Neurol Surg Rep 2021;82:e32-e35.
Address for correspondence Daryoush Tavanaiepour, MD, Department of Neurosurgery, University of Florida, College of Medicine, 580 West 8th Street, 8th floor, Clinical Tower 1, Jacksonville, FL 32209, United Sates (e-mail: daryoush.tavanaiepour@jax.ufl.edu).

\begin{abstract}
Keywords

- falx cerebelli

- meningioma

- posterior fossa

- pseudomeningocele
\end{abstract}

The falx cerebelli is a small crescent fold of dura mater that is attached to the internal occipital crest and projects forward into the posterior cerebellar notch between the cerebellar hemispheres. We report a rare case of a 61-year-old female who presented with a 1-month history of headache and gait instability. Imaging findings were suggestive of a meningioma arising from the falx cerebelli. Complete surgical resection was achieved with a standard posterior fossa midline approach. Duraplasty was performed using animal allograft dura (Duraguard) and additional layers of oxidized cellulose preparation (Surgicel), fibrin sealant, and nonsuturable collagen matrix (Duragen) were utilized to reduce the risk of a cerebrospinal fluid leak. Pathology confirmed a World Health Organization (WHO) grade-I meningioma. Postoperatively, patient with asymptomatic thrombosis of the left transverse/sigmoid sinuses and later with a pseudomeningocele managed with a lumbar drain. To our knowledge, this is the second documented case in the literature. We discuss intraoperative nuances and unique aspects in the postoperative care and management of these patients.

\section{Introduction}

The falx cerebelli is a small crescentic fold of dura mater that is attached to the internal occipital crest and projects forward into the posterior cerebellar notch between the cerebellar hemispheres. ${ }^{1}$ The posterior margin contains the occipital sinus, and it is attached to the lower surface of the tentorium cerebelli. ${ }^{2}$ We report a case of a falx cerebelli meningioma that to our knowledge is the second documented case in the English literature.

\section{Case Report}

A 61-year-old female presented with a 1-month history of headache with increase in severity over a period of a week. Patient also reported symptoms of intermittent dizziness, blurred vision, and gait instability. Neurological examination revealed dysmetria of the right hand. Computed tomography demonstrated a midline cerebellar mass and mass effect on the fourth ventricle with partial effacement. There was no evidence of hydrocephalus. Magnetic resonance imaging received

October 3, 2020

accepted after revision

May 26, 2021
DOI https://doi.org/ $10.1055 / \mathrm{s}-0041-1735905$. ISSN 2193-6358.

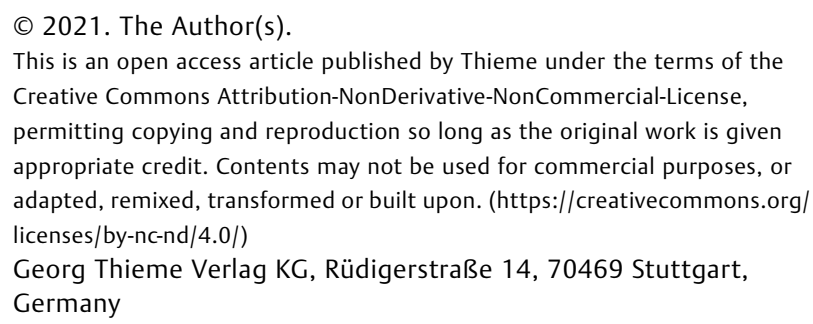




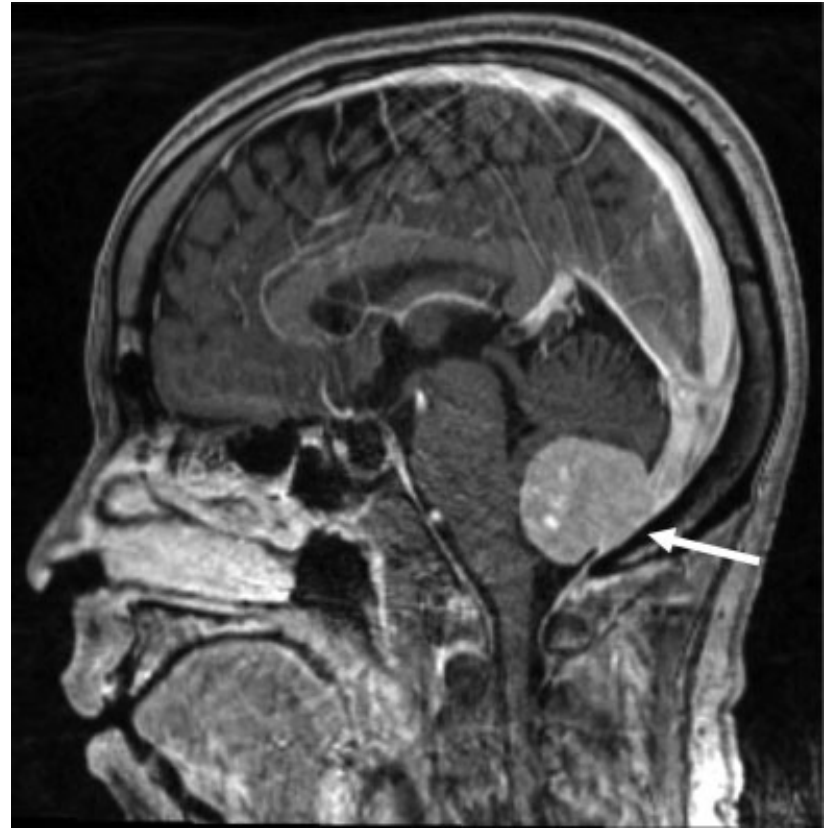

Fig. 1 Sagittal enhanced T1 MRI of the brain demonstrating a relatively homogenously enhancing mass lesion with dural attachment to the falx cerebelli and occipital sinus invasion (arrow). MRI, magnetic resonance imaging.

(MRI) revealed a homogenously enhancing solid mass within the midinferior vermis with a broad dural attachment (-Fig. 1). Furthermore, the occipital sinus was prominent with dominant bilateral transverse sinuses that were patent (-Fig. 2). Due to the tumor size, mass effect on posterior fossa structures and clinical progression of

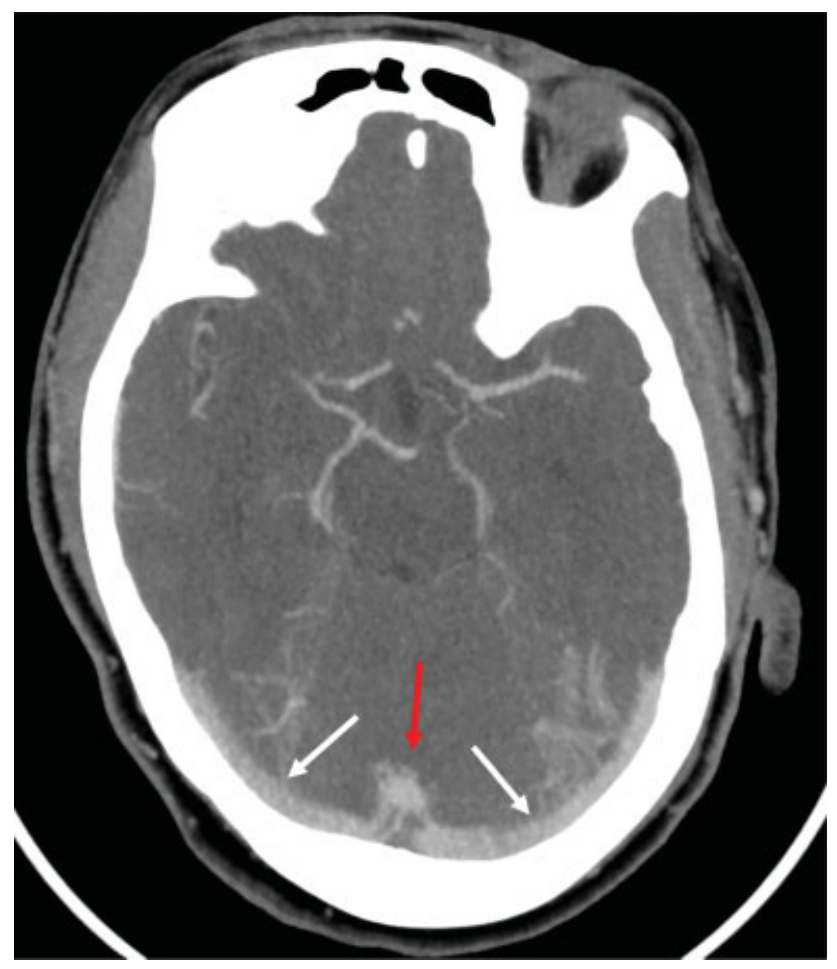

Fig. 2 Axial CT venogram image demonstrating patent dominant bilateral transverse sinuses (white arrows) and prominent occipital sinus (red arrow). CT, computed tomography. symptomatic brainstem compression, we recommended surgical intervention and patient consent was obtained.

Patient was placed in prone position and a standard midline occipital craniectomy was performed. ${ }^{3}$ The dura was opened in a semilunar fashion with the base against the occipital sinus, both on the left and right side of the occipital sinus. Microsurgical technique was used to perform arachnoid dissection of the bilateral hemispheres, exposing the tumor in its entirety. Next, the tumor was debulked. Direct visualization confirmed that the cerebelli falx was the origin of the tumor attachment and vascular supply. The occipital sinus above and below the tumor was ligated and the falx cerebelli was completely excised. This then allowed for complete en bloc resection of the tumor including its dural attachments. Due to the risk of cerebrospinal fluid (CSF) leak ${ }^{4}$ duraplasty was performed by placing animal allograft dura (Duraguard) in an inlaid gasket fashion and circumferentially placed sutures. Then, an oxidized cellulose preparation (Surgicel) was placed along the suture sites with fibrin sealant and a nonsuturable collagen matrix (Duragen) on top. A mesh cranioplasty with bone substitute was placed.

Pathology confirmed a World Health Organization (WHO) grade-I meningioma. Tumor histology was characterized by transitional and fibroblastic cell patterns of meningioma (epithelial membrane antigen positive) with psammomatous calcification and small few foci of microcystic to clear cell changes ( $\mathbf{- F i g . 3}$ ). Mitoses were inconspicuous and $\mathrm{K}_{\mathrm{i}}-67$ proliferation marker labeled less than $5 \%$ of tumor cells. No atypical cellular features or tumor necrosis were present. Brain tissue was focally present at the tumor periphery with no features of brain invasion by the tumor.

Postoperatively, patient had no neurologic deficits. Imaging revealed a gross total resection ( - Fig. 4) and an asymptomatic thrombosis of the left transverse/sigmoid sinuses (-Fig. 5). This was treated conservatively with intravenous fluids and close observation. Nine days following resection, patient had a pseudomeningocele that was treated with lumbar drain placement for 3 days.

At her 2-week postoperative wound check, patient's incision was well healed and with minimal headaches which resolved by the next office visit, 6 weeks after surgery. Given gross total resection and WHO grade- 1 pathology, patient was monitored with serial imaging. Resolution of pseudomeningocele was noted on MRI completed 3 months after surgery. Almost 1 year after surgery, there is no evidence of tumor recurrence and almost fully recanalization of the left transverse/sigmoid sinuses.

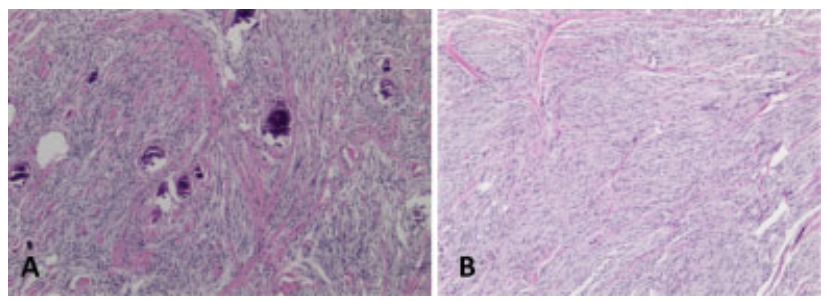

Fig. 3 Transitional (A) and spindle cell fibroblastic (B) patterns of meningioma (HE stain $\times 5$ ). HE, hematoxylin and eosin. 


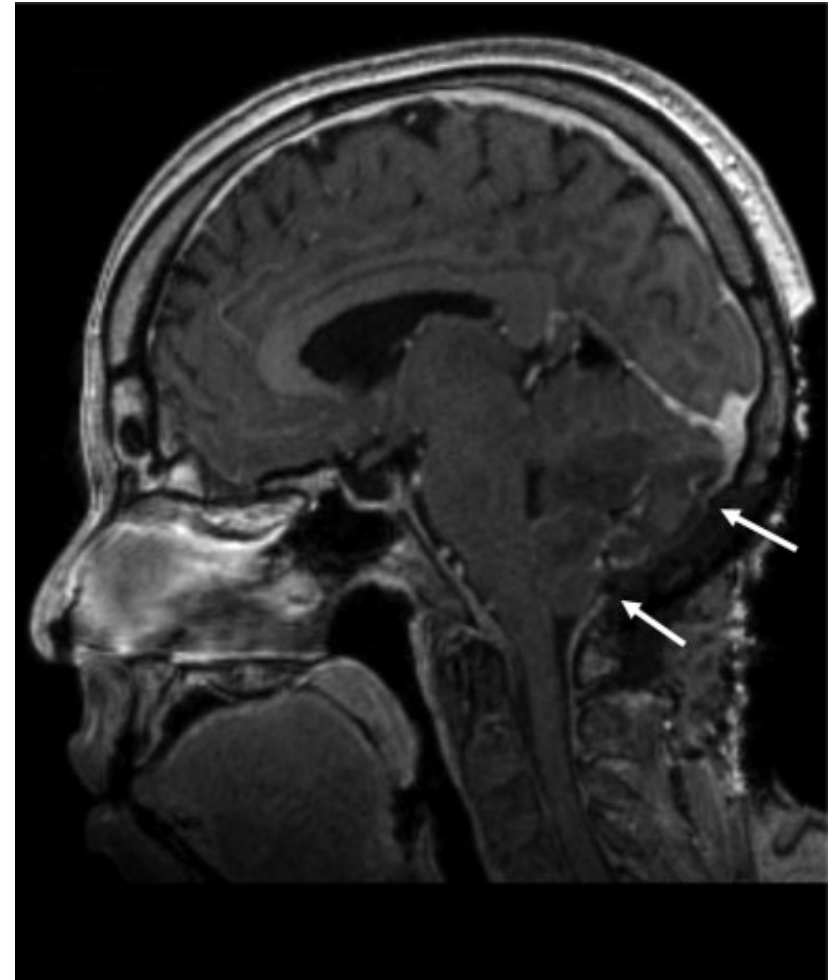

Fig. 4 Sagittal enhanced T1 MRI of the brain demonstrating gross total resection including the falx cerebelli above and below the tumor (arrows). MRI, magnetic resonance imaging.

\section{Discussion}

Posterior fossa meningiomas make up to $9 \%$ of all the intracranial meningiomas. ${ }^{5}$ They either involve cerebellar convexity or lateral convexity meningiomas. To our knowledge, this is the second report of a falx cerebelli meningioma. ${ }^{6}$ The MRI features of a falx cerebelli meningioma can be similar to that of other types of tumors, making it difficult to differentiate tumors based on radiological findings alone. ${ }^{7}$ Falx cerebelli meningiomas are very analogous to cerebral falcine meningiomas. The latter are described as arising from the cerebral falx concealed completely by overlying cortex. They have a dumbbell shape and invaginate into the medial aspects of both left and right hemispheres and in some patients, the tumor grows into the inferior edge of the sagittal sinus. ${ }^{1}$ This current rare case of a falx cerebelli meningioma had all the features of a falcine meningioma.

In this type of case, dural venous anatomy requires careful consideration. The standard midline suboccipital approach entails ligation of the occipital sinus. ${ }^{3}$ The occipital sinus is the smallest of the sinuses and lies in the attached margin of the falx cerebelli. ${ }^{8}$ However, there are existing variations of the dural sinuses that can prove hazardous with this approach. $^{8-10}$ In this case, there was a prominent occipital sinus. Several studies present that some occipital sinuses function as the main drainage route of the intracranial veins instead of the transverse sinus or sigmoid sinus. ${ }^{8}$ In this patient's case, ligation of the occipital sinus was deemed safe because of the bilateral dominant transverse sinuses.

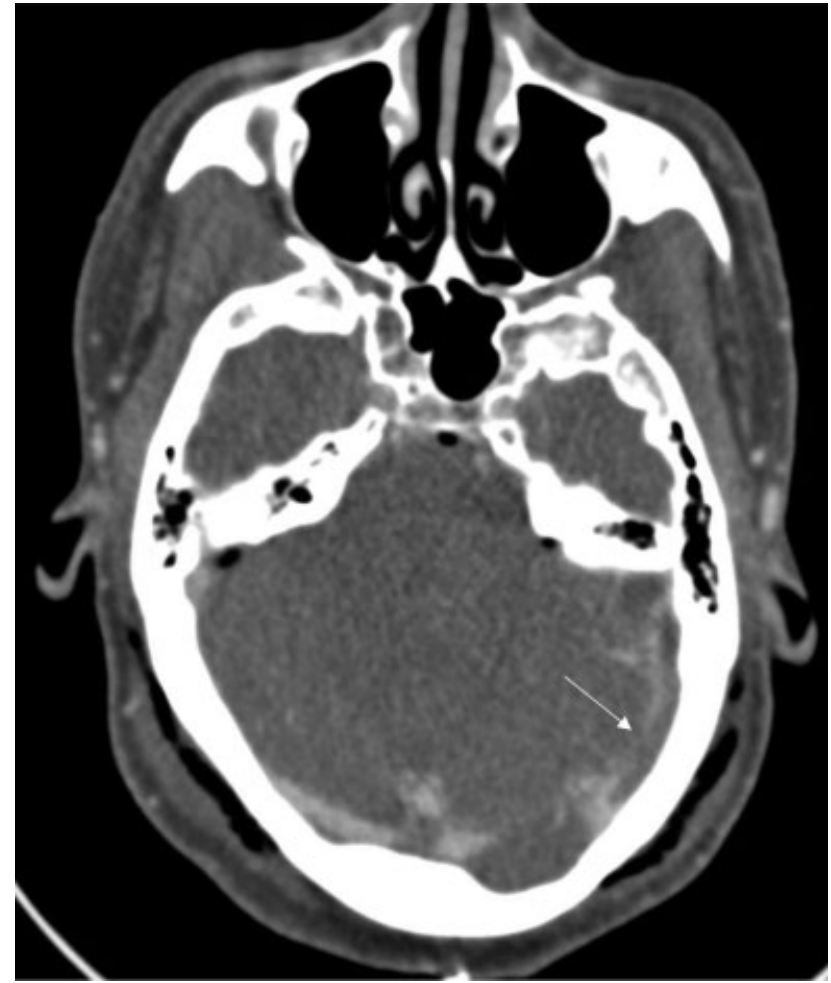

Fig. 5 Axial CT venogram image demonstrating a filling defect consistent with thrombus in the left transverse and sigmoid sinuses (arrow). CT, computed tomography.

While the suboccipital midline approach is common for posterior fossa lesions, it can lead to complications. Major postoperative complications include pseudomeningocele, CSF leak, and sinus thrombosis. ${ }^{11,12}$ Postoperative transverse or sigmoid sinus thrombosis has been reported to occur in 4 to $11 \%$ of cases following posterior fossa surgery. ${ }^{13}$ Theories regarding related risk factors for postoperative sinus thrombosis point to intraoperative manipulation or retraction of the sinus $^{14}$; however, in this case, the left transverse/sigmoid sinus was not exposed during the midline approach. Furthermore, ligation of the occipital sinus is unlikely to be the cause of the patient's thrombosis as it occurred distally to the ligation as opposed to proximally. Given the risk of postoperative intracranial hemorrhage ${ }^{15}$ and lack of clear guidelines on how to treat an asymptomatic postoperative sinus thrombosis, we opted to treat conservatively with intravenous (IV) fluids and subcutaneous heparin as per the standard of care at our institution for deep venous thrombosis prophylaxis.

The rate of pseudomeningocele from posterior fossa surgery ranges from 4 to $28 \%$ and CSF leak ranges from 2 to $23 \%{ }^{12}$ Postoperative posterior fossa pseudomeningoceles can be related to underlying hydrocephalus, ligation of the occipital sinus, thrombosis of the sinuses, and other factors such as difficult dural closure. ${ }^{3,12,16}$ However, there are various dural substitutes, sealants, and modified surgical techniques that can be utilized to obtain a watertight dura closure to help prevent postoperative pseudomeningocele. ${ }^{12}$ In this case, animal allograft, a nonsuturable collagen matrix, and fibrin sealant were used to lower the risk of CSF leak. 
While our patient was found to have a pseudomeningocele despite the use of several barriers, it resolved with conservative first-line treatment of lumbar drainage ${ }^{4}$ for 3 days.

\section{Conclusion}

To our knowledge, this is the second reported case of a falx cerebelli meningioma. Using standard posterior fossa midline approach, gross total resection of the tumor was achieved without neurological deficits. A prominent occipital sinus was ultimately deemed safe to ligate due to dominant bilateral transverse sinuses. Postoperatively, patient developed an asymptomatic thrombosis of the left transverse/sigmoid sinuses and a pseudomeningocele which responded well to conservative management. Falx cerebelli meningiomas are very analogous to cerebral falcine meningiomas, in terms of their radiological and intraoperative features. In this particular case, gross total resection of falx cerebelli meningioma was feasible.

\section{Conflict of Interest}

None declared.

\section{References}

1 Mavridis IN, Lontos K, Anagnostopoulou S. Absent falx cerebelli: report of a rare case. Anat Sci Int 2013;88(03):181-182

2 Mooney MA, Abolfotoh M, Bi WL, et al. Is falcine meningioma a diffuse disease of the falx? Case series and analysis of a "grade zero" resection. Neurosurgery 2020;87(05):900-909

3 Panigrahi M, Samiruddin SM, Vooturi S. Crescent durotomy for midline posterior fossa lesions. Neurol India 2019;67(01):155-158

4 Smith GA, Strohl MP, Manjila S, Miller JP. Incidence, management, and outcome of symptomatic postoperative posterior fossa pseudomeningocele: a retrospective single-institution experience. Oper Neurosurg (Hagerstown) 2016;12(03):298-304
5 Javalkar V, Banerjee AD, Nanda A. Posterior cranial fossa meningiomas. Skull Base 2012;73(Suppl 1):1-10

6 Agrawal A. Falx cerebelli meningioma: technical note. Romanian Neurosurgery 2014;21(03):337-340

7 Ito M, Kamiyama H, Nakamura T, Nakajima H, Tokugawa J. Dural cavernous hemangioma of the cerebellar falx. Neurol Med Chir (Tokyo) 2009;49(09):410-412

8 Shin HS, Choi DS, Baek HJ, et al. The oblique occipital sinus: anatomical study using bone subtraction 3D CT venography. Surg Radiol Anat 2017;39(06):619-628

9 Candanedo C, Moscovici S, Kaye AH, Spektor S. The oblique occipital sinus - implications in posterior fossa approaches. J Clin Neurosci 2020;76:202-204

10 Hamnett NTJ, Ogungbo B, Nahser H, Javadpour M. Anomalous cerebral venous sinus drainage. Br J Neurosurg 2010;24(04): 497-498

11 Abou-Al-Shaar H, Gozal YM, Alzhrani G, Karsy M, Shelton C, Couldwell WT. Cerebral venous sinus thrombosis after vestibular schwannoma surgery: a call for evidence-based management guidelines. Neurosurg Focus 2018;45(01):E4

12 Zhao Y, Chen L, Zhang J, et al. Duraplasty with cervical fascia autograft to reduce postoperative complications of posterior fossa tumor surgery with suboccipital midline approach. World Neurosurg 2020;134:e1115-e1120

13 Orlev A, Jackson CM, Luksik A, et al. Natural history of untreated transverse/sigmoid sinus thrombosis following posterior fossa surgery: case series and literature review. Oper Neurosurg (Hagerstown) 2020;19(02):109-116

14 Benjamin CG, Sen RD, Golfinos JG, et al. Postoperative cerebral venous sinus thrombosis in the setting of surgery adjacent to the major dural venous sinuses. J Neurosurg 2018;131(04):1-7

15 Apra C, Kotbi O, Turc G, et al. Presentation and management of lateral sinus thrombosis following posterior fossa surgery. J Neurosurg 2017;126(01):8-16

16 Higgins JN, Macfarlane R, Axon PR, et al. Headache, cerebrospinal fluid leaks, and pseudomeningoceles after resection of vestibular schwannomas: efficacy of venous sinus stenting suggests cranial venous outflow compromise as a unifying pathophysiological mechanism. J Neurol Surg B Skull Base 2019;80(06):640-647 